\title{
New kinematic distance scale for the Galactic planetary nebulae
}

\author{
Alexander F. Kholtygin ${ }^{1}$, Igor' I. Nikiforov ${ }^{1}$ and Vitalii V. Akimkin ${ }^{2}$ \\ ${ }^{1}$ Sobolev Astronomical Institute, Saint Petersburg State University, \\ Universitetskij pr. 28, Staryj Peterhof, Saint Petersburg 198504, Russia \\ email: afkholtygin@gmail.com \\ ${ }^{2}$ Astronomical Institute of Russian Academy of Science, Russia
}

\begin{abstract}
We analyze the kinematics of disk planetary nebulae (PNe) to derive the formal Galactic center distance, $R_{0}$, for three catalogues of PNe distances. Then we correct the catalogues' distance scales renormalizing the PNe distances by the ratios of formal $R_{0}$ values to a best modern value of $R_{0}=7.9 \mathrm{kpc}$. The created new catalogue of disk's PNe distances was found to be in a good agreement with distances by Stanghellini et al. (2008). Our catalogue of $\mathrm{PNe}$ distances was used to recalibrate the statistical distance scale for all Galactic PNe.
\end{abstract}

Keywords. Planetary nebulae: general, Galaxy: kinematics and dynamics

\section{Kinematic modelling and renormalization of PNe distances}

In this paper we use the method of kinematic modelling $\mathrm{PNe}$ ensemble by Nikiforov \& Bobrova (1999) (see details also in Nikiforov 1999, see preliminary results in Kholtygin et al. 2010). With the assumption of axisymmetric rotation, the heliocentric radial velocity $V_{r}$ of an object of a disk subsystem, can be represented by the following model:

$$
V_{r, \bmod }=\left[-2 A\left(R-R_{0}\right)+\sum_{i=2}^{n} \theta_{i}\left(R-R_{0}\right)^{i}\right] \frac{R_{0}}{R} \sin l \cos b-\mathbf{v}_{0},
$$

where $R$ is the distance to the Galactic axis, $R_{0}$ is the Sun-Galactic center distance; $l$ and $b$ are the Galactic coordinates of the object; $\mathbf{v}_{0}$ is the contribution of the solar motion relative to the system of objects $(\mathrm{PNe}) ; K$ is the $k$-term; $A$ is the Oort constant.

For each catalogue of PNe distances, we derive the set of optimal model parameters $R_{0}$, $A, \theta_{2}, \ldots, \theta_{n}, \mathbf{v}_{0}, K$ by the nonlinear least-square fit; for every sample of $\mathrm{PNe}$, the optimal degrees $n$ is also determined. An obtained formal $R_{0}$ value, $R_{0}^{(\mathrm{f})}$, is used to correct the scale of distances to PNe in the catalogue. The correction factor is $G_{\text {scale }}=R_{0}^{(\mathrm{b})} / R_{0}^{(\mathrm{f})}$, where $R_{0}^{(\mathrm{b})}=7.9 \pm 0.2 \mathrm{kpc}$ is the current "best estimation" of $R_{0}$ (Nikiforov 2004). The corrected distances $r_{i}^{\text {corr }}$ to the $i$-th PN can be calculated as $r_{i}^{\text {corr }}=G_{\text {scale }} r_{i}$.

In Fig. 1 we compare our and Stanghellini et al. (2008) distances for common objects. Overall we see a good agreement between the scales, but for PNe with $r<3 \mathrm{kpc}$ the distances by Stanghellini et al. (2008) are 15-20\% lower then ours (Fig. 1, right panel).

We also find a good agreement between our data and the most of individual distances (Harris et al. 2007; Stanghellini et al. 2008).

\section{New calibration of the distance scale for $\mathrm{PNe}$}

The optimal parameters and correction factors $G_{\text {scale }}$ for the catalogues considered are presented in Table 1. The corrected distances were determined for more than $300 \mathrm{PNe}$.

The statistical distances by Stanghellini et al. (2008) are based on a calibration of relation between the ionized mass of $\mathrm{PN} \mu=1.505 \times 10^{-3} r^{5 / 2} \vartheta^{3 / 2} F^{1 / 2}$ and the optical 
Table 1. Parameters of kinematic models for different ensembles of PNe.

\begin{tabular}{lccccc}
\hline Catalogue & Number of PNe & $n_{\mathrm{opt}}$ & $A, \mathrm{~km} \mathrm{~s}^{-1} \mathrm{kpc}^{-1}$ & $\left\langle R_{0}\right\rangle, \mathrm{kpc}$ & $G_{\text {scale }}$ \\
\hline Acker (1978) & 233 & 1 & $15.5 \pm 1.2$ & $5.39 \pm 0.48$ & $1.47 \pm 0.06$ \\
Cahn et al. $(1992)$ & 277 & 2 & $12.6 \pm 1.0$ & $5.65 \pm 0.44$ & $1.40 \pm 0.05$ \\
Phillips (2004) & 219 & 1 & $12.3 \pm 0.8$ & $6.70 \pm 0.51$ & $1.16 \pm 0.06$ \\
\hline
\end{tabular}
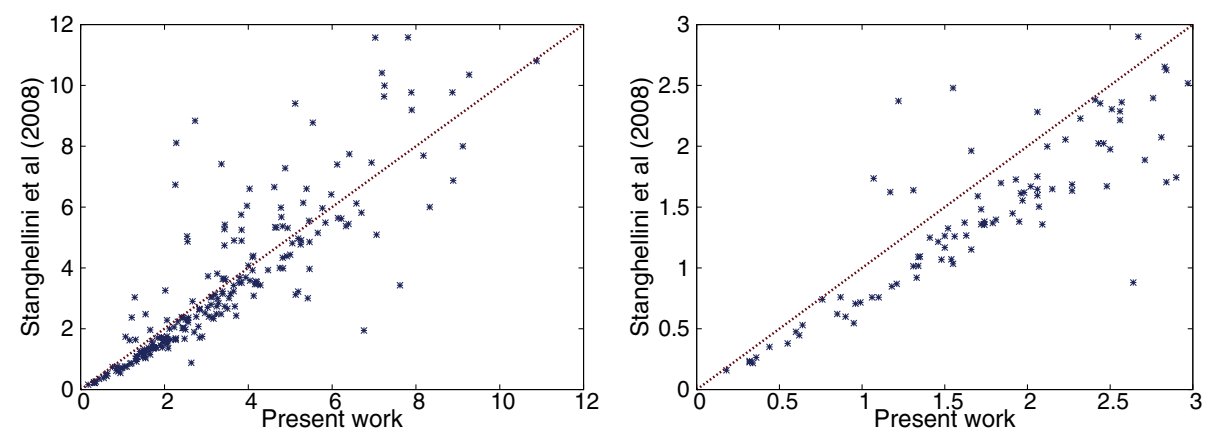

Figure 1. Left: A comparison between distances $r$ to PNe (in kpc) obtained in this paper and in Stanghellini et al. (2008). Right: The same as in the left panel, but for $r<3 \mathrm{kpc}$.

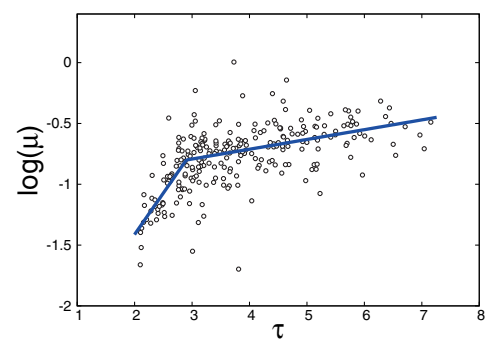

Figure 2. The parameter $\log \mu$ vs. $\tau$ for kinematically calibrated distances. The empty circles mark individual nebulae, the solid line shows the approximation (2.1).

thickness parameter $\tau=\log \left(4 \vartheta^{2} / F\right)$. Here $r$ is the distance to an PN in parsecs, $\vartheta$ is the nebular radius in arcseconds, and $F$ is the nebular flux at $5 \mathrm{GHz}$. The best fit to the distance scale based on our kinematically calibrated PNe distances is as follows:

$$
\log \mu= \begin{cases}0.08 \tau-1.04, & \tau \geqslant 2.89 \\ 0.69 \tau-2.80, & \tau \leqslant 2.89\end{cases}
$$

In Fig. 2 we demonstrate the quality of the fit (2.1). This fit gives us a better agreement with the individual distances to PNe than any other statistical scale.

\section{References}

Acker, A. 1978, A\& AS, 33, 367

Cahn, J. H., Kaler, J. B., Stanghellini, L., et al. 1992, Aछ̈AS, 94, 399

Harris, H. C., Danh, C. C., Canzian, B., et al. 2007, AJ, 133, 631

Kholtygin, A. F., Milanova, Yu. V., \& Akimkin, V. V. 2010, Highlights of Astronomy, 15, 792

Nikiforov, I. I. 1999, Astrophysics, 42, 300

Nikiforov, I. I. \& Bobrova (Mel'nichnikova), A. Yu. 1999, Kinem. Phys. Cel. Bodies, appl. 2, 29

Nikiforov I. I. 2004, in: G. G. Byrd, K. V. Kholshevnikov, A. A. Mylläri, I. I. Nikiforov, \& V. V.

Orlov (eds.), Order and Chaos in Stellar and Planetary Systems, ASP Conf. Ser., 316, 199

Phillips, J. P. 2004, MNRAS, 353, 589

Stanghellini, L., Shaw, R. A., \& Villaver, E. 2008, ApJ, 689, 194 